\title{
Palladium-catalyzed Heck-type reaction of secondary trifluoromethylated alkyl bromides
}

\author{
Tao Fan ${ }^{1}$, Wei-Dong Meng ${ }^{1}$ and Xingang Zhang ${ }^{* 2}$
}

\author{
Full Research Paper \\ Address: \\ ${ }^{1}$ College of Chemistry, Chemical Engineering and Biotechnology, \\ Donghua University, 2999 North Renmin Road, Shanghai 201620, \\ China, and ${ }^{2}$ Key Laboratory of Organofluorine Chemistry, Shanghai \\ Institute of Organic Chemistry, Chinese Academy of Sciences, 345 \\ Lingling Road, Shanghai 200032, China \\ Email: \\ Xingang Zhang ${ }^{*}$ - xgzhang@mail.sioc.ac.cn. \\ * Corresponding author \\ Keywords: \\ alkenes; cross-coupling; Heck-type reaction; palladium; secondary \\ trifluoromethylated alkyl bromides \\ Beilstein J. Org. Chem. 2017, 13, 2610-2616. \\ doi:10.3762/bjoc. 13.258 \\ Received: 30 September 2017 \\ Accepted: 21 November 2017 \\ Published: 06 December 2017 \\ This article is part of the Thematic Series "Organo-fluorine chemistry IV". \\ Guest Editor: D. O'Hagan \\ (C) 2017 Fan et al.; licensee Beilstein-Institut. \\ License and terms: see end of document.
}

\begin{abstract}
An efficient palladium-catalyzed Heck-type reaction of secondary trifluoromethylated alkyl bromides has been developed. The reaction proceeds under mild reaction conditions with high efficiency and excellent functional group tolerance, even towards formyl and hydroxy groups. Preliminary mechanistic studies reveal that a secondary trifluoromethylated alkyl radical is involved in the reaction.
\end{abstract}

\section{Introduction}

With the increasing number of applications of fluorinated compounds in life and materials science, developing efficient and straightforward methods for the synthesis of fluorinated compounds has become more and more important. Although great achievements have been made in the introduction of fluorine atom(s) into organic molecules over the past decade, investigations mainly focused on direct fluorination or fluoroalkylation of aromatics [1-5]. Fluoroalkylated alkenes are a kind of important fluorinated structural motifs and have wide applications in medicinal chemistry and advanced functional materials [6-9]. However, developing efficient methods for the synthesis of such valuable structures has received less attention [10-15].

Commonly, fluoroalkylated alkenes can be prepared through fluoroalkyl radical addition of alkynes via an atom transfer pathway [16,17] or cross-coupling of alkenyl halides with fluoroalkyl metal species [18-24]. Recently, we reported a palladium-catalyzed Heck-type reaction of fluoroalkyl bro- 
mides, representing an efficient and straightforward alternative to access fluoroalkylated alkenes [25-27]. Preliminary mechanistic studies reveal that a fluoroalkyl radical via a single electron transition (SET) pathway is involved in the reaction. Inspired by this work, we question that whether secondary fluoroalkylated alkyl bromides are also suitable substrates for such a Heck-type reaction. To the best of our knowledge, the palladium-catalyzed Heck-type reaction of secondary fluoroalkylated alkyl bromides with alkenes remains a challenge and has not been reported so far [28-32] due to the sluggish oxidative addition [33] of alkyl halides to palladium and facile $\beta$-hydride elimination $[34,35]$ of alkylpalladium species. Additionally, the resulting fluoroalkylated allylic compounds can serve as a versatile building block for the synthesis of complex fluorinated molecules [36,37]. Herein, we describe a palladiumcatalyzed Heck-type reaction of secondary trifluoromethylated alkyl bromides. The reaction proceeds under mild reaction conditions with broad substrate scope and high efficiency. The reaction can also extend to secondary difluoroalkylated alkyl iodide. Preliminary mechanistic studies reveal that a secondary alkyl radical via a SET pathway is involved in the reaction.

\section{Results and Discussion}

We began this study by choosing styrene (1a) and 2-bromo1,1,1-trifluorohexane (2a) as model substrates (Table 1).
Initially, a 27\% yield of product 3a along with small amount of hydrodebrominated byproduct $\mathbf{3} \mathbf{a}^{\prime}$ ( $2 \%$ yield) were obtained when the reaction of $\mathbf{1 a}(0.2 \mathrm{mmol}, 1.0$ equiv $)$ with $\mathbf{2 a}$ (2.0 equiv) was carried out in the presence of $\mathrm{PdCl}_{2}\left(\mathrm{PPh}_{3}\right)_{2}$ ( $5 \mathrm{~mol} \%$ ), Xantphos (10 mol \%) and $\mathrm{K}_{2} \mathrm{CO}_{3}$ (2.0 equiv) in DCE for $16 \mathrm{~h}$ at $80^{\circ} \mathrm{C}$ (Table 1, entry 1). After optimization of the reaction conditions (for details, see Supporting Information File 1), a dramatically improved yield of 3a (83\% yield upon isolation) was provided by using KOAc as a base (Table 1 , entry 5). $\mathrm{K}_{3} \mathrm{PO}_{4}$ also led to a good yield of 3a (Table 1, entry 6), but other bases, such as $\mathrm{Na}_{2} \mathrm{CO}_{3}, \mathrm{Cs}_{2} \mathrm{CO}_{3}, \mathrm{NaOAc}$ and $\mathrm{KF}$ were less effective (Table 1, entries 2-4 and 7). Among all the tested palladium salts (Table 1, entries 8-12), $\mathrm{PdCl}_{2}\left(\mathrm{PPh}_{3}\right)_{2}$ was proved to be the most effective catalyst and provided 3a in $84 \%$ yield (Table 1, entry 5). The use of Xantphos was crucial for the reaction efficiency (Table 1, entry 5). Other ligands either led to low yield or showed no reactivity (Table 1, entry 13, for details, see Supporting Information File 1). The beneficial effect of Xantphos is probably due to its large bit angle to promote the reaction $[38,39]$. However, the exact role of Xantphos remains elusive. Finally, the optimal reaction conditions were identified by decreasing the loading amount of Xantphos from $10 \mathrm{~mol} \%$ to $7.5 \mathrm{~mol} \%$ with a higher concentration of $1 \mathbf{a}$ and $\mathbf{2 a}$, providing $\mathbf{3 a}$ in $88 \%$ yield upon isolation (Table 1 , entry 14).

Table 1: Representative results for the optimization of Pd-catalyzed cross-coupling between 1a and 2-bromo-1,1,1-trifluorohexane (2a)

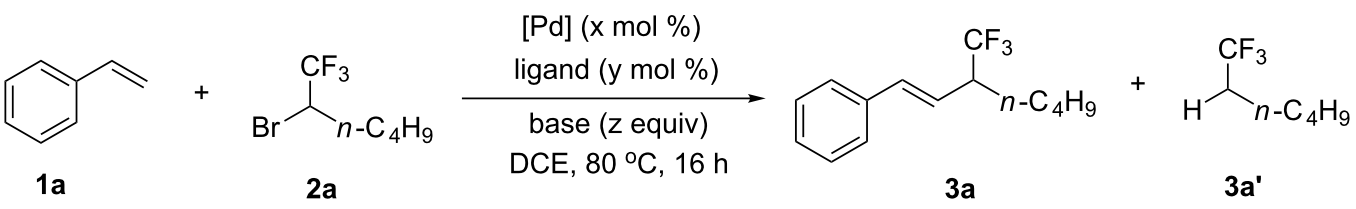

\begin{tabular}{|c|c|c|c|c|}
\hline entry & {$[\mathrm{Pd}](\mathrm{mol} \%)$} & ligand $(\mathrm{mol} \%)$ & base (equiv) & $3 \mathbf{a}$, yield $(\%)^{b}$ \\
\hline 1 & $\mathrm{PdCl}_{2}\left(\mathrm{PPh}_{3}\right)_{2}(5)$ & Xantphos (10) & $\mathrm{K}_{2} \mathrm{CO}_{3}(2)$ & 27 \\
\hline 2 & $\mathrm{PdCl}_{2}\left(\mathrm{PPh}_{3}\right)_{2}(5)$ & Xantphos (10) & $\mathrm{Na}_{2} \mathrm{CO}_{3}(2)$ & 5 \\
\hline 3 & $\mathrm{PdCl}_{2}\left(\mathrm{PPh}_{3}\right)_{2}(5)$ & Xantphos (10) & $\mathrm{Cs}_{2} \mathrm{CO}_{3}(2)$ & 26 \\
\hline 4 & $\mathrm{PdCl}_{2}\left(\mathrm{PPh}_{3}\right)_{2}(5)$ & Xantphos (10) & $\mathrm{NaOAc}(2)$ & 42 \\
\hline 5 & $\mathrm{PdCl}_{2}\left(\mathrm{PPh}_{3}\right)_{2}(5)$ & Xantphos (10) & KOAc (2) & $84(83)$ \\
\hline 6 & $\mathrm{PdCl}_{2}\left(\mathrm{PPh}_{3}\right)_{2}(5)$ & Xantphos (10) & $\mathrm{K}_{3} \mathrm{PO}_{4}(2)$ & 75 \\
\hline 7 & $\mathrm{PdCl}_{2}\left(\mathrm{PPh}_{3}\right)_{2}(5)$ & Xantphos (10) & KF (2) & 37 \\
\hline 8 & $\mathrm{PdCl}_{2}(5)$ & Xantphos (10) & KOAc (2) & 18 \\
\hline 9 & $\mathrm{Pd}(\mathrm{OAc})_{2}(5)$ & Xantphos (10) & KOAc (2) & 51 \\
\hline 10 & $\mathrm{PdCl}_{2} \cdot \mathrm{dppp}(5)$ & Xantphos (10) & KOAc (2) & Trace \\
\hline 11 & $\mathrm{PdCl}_{2} \cdot \mathrm{dppf}(5)$ & Xantphos (10) & KOAc (2) & 69 \\
\hline 12 & $\mathrm{Pd}\left(\mathrm{PPh}_{3}\right)_{4}(5)$ & Xantphos (10) & $\mathrm{KOAc}(2)$ & 64 \\
\hline 13 & $\mathrm{PdCl}_{2}\left(\mathrm{PPh}_{3}\right)_{2}(5)$ & dprephos & KOAc (2) & 45 \\
\hline $14^{\mathrm{C}}$ & $\mathrm{PdCl}_{2}\left(\mathrm{PPh}_{3}\right)_{2}(5)$ & Xantphos (7.5) & KOAc (2) & $95(88)$ \\
\hline
\end{tabular}

aReaction conditions (unless otherwise specified): 1 a $(0.2 \mathrm{mmol}, 1.0$ equiv), $2 \mathrm{a}$ ( 2.0 equiv), base (2.0 equiv), DCE ( $3 \mathrm{~mL}), 16 \mathrm{~h} .{ }^{\mathrm{b}} \mathrm{Determined} \mathrm{by}$ ${ }^{19} \mathrm{~F} \mathrm{NMR}$ using fluorobenzene as an internal standard (isolated yield in parentheses). ${ }^{\mathrm{c}} \mathbf{1 a}(0.4 \mathrm{mmol}, 1.0$ equiv), $2 \mathrm{a}(2.0$ equiv) and DCE (3 mL) were used. 
With the optimized reaction conditions in hand, a variety of alkenes were examined. As shown in Scheme 1, reactions of $\mathbf{2 a}$ with a series of styrene derivatives $\mathbf{1}$ proceeded smoothly and provided $\mathbf{3}$ in moderate to excellent yields (Scheme 1). Generally, substrates bearing electron-donating groups afforded higher yields than that of alkenes bearing electron-withdrawing groups. Various versatile functional groups, such as ester, cyano, and chloride showed good tolerance to the reaction $(\mathbf{3 f}-\mathbf{j})$. Vinylnaphthalene also furnished the corresponding product efficiently (3k). The conjugated alkene did not interfere with the reaction efficiency, providing $\mathbf{3 1}$ in $83 \%$ yield. The reaction was not restricted to aromatic alkenes, enamide was also applicable to the reaction and afforded $3 \mathrm{~m}$ in $61 \%$ yield.

To demonstrate the generality of this method further, reactions of alkenes with various secondary trifluoromethylated alkyl bromides were performed and provided the corresponding products 4 with high yield. As shown in Scheme 2, a variety of secondary trifluoromethylated alkyl bromides, including those sub- strates bearing base and nucleophile sensitive functional groups, such as hydroxy, aldehyde, and phthalimide (4e, $\mathbf{4 f}, \mathbf{4 j}$ and $\mathbf{4 k}$ ), were all applicable to the reaction, thus offering good opportunities for downstream transformations and highlighting the utility of the current process further. (3-Bromo-4,4,4-trifluorobutyl)benzene and 4-bromo-5,5,5-trifluoropentyl benzoate were also successfully employed to couple with a conjugated alkene and afforded the corresponding products $4 \mathbf{d}$ and $\mathbf{4 h}$ in good yields. The current cross coupling was also applicable to the secondary difluoroalkylated alkyl halides as demonstrated by the representative reaction of secondary ethyl 2,2-difluoro-2iodoacetate with para-methoxystyrene and para-fluorostyrene (4I and $4 \mathrm{~m})$.

To probe whether a secondary trifluoromethylated alkyl radical is involved in the current reaction, radical inhibition experiments were performed (Table 2). When a reaction of $\mathbf{1 a}$ with $\mathbf{2 a}$ was carried out in the presence of $\mathrm{PdCl}_{2}\left(\mathrm{PPh}_{3}\right)_{2}(5 \mathrm{~mol} \%)$, Xantphos $(7.5 \mathrm{~mol} \%)$ and KOAc in DCE at $80^{\circ} \mathrm{C}$, the addition

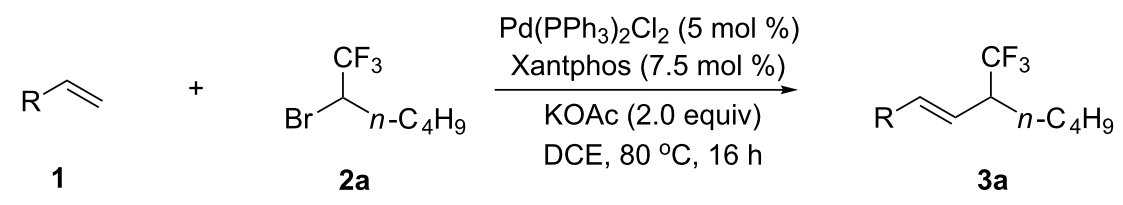<smiles>CCOC(/C=C/c1ccccc1)C(F)(F)F</smiles>

3a, $88 \%$<smiles>CCCCCCC(=CC=Cc1ccc(C)cc1)C(F)(F)F</smiles>

3b, $91 \%$<smiles>CCCCCCC(=CC=Cc1ccccc1C)C(F)(F)F</smiles>

3c, $90 \%$<smiles>CCCCCCC(=CC=Cc1ccc(C(C)(C)C)cc1)C(F)(F)F</smiles>

3d, $90 \%$<smiles>CCCCCCC(/C=C/c1ccc(OC)cc1)C(F)(F)F</smiles>

3e, $97 \%$<smiles>CCOC(/C=C/c1ccc(OC(C)=O)cc1)C(F)(F)F</smiles>

3f, $95 \%$<smiles>CCCCCCCC(=CC=Cc1ccc(C(C)=O)cc1)C(F)(F)F</smiles>

3g, $63 \%$<smiles>CCCCCC(=CC=Cc1ccc(C#N)cc1)C(F)(F)F</smiles>

3h, $72 \%$<smiles>[X]c1ccc(/C=C/C(OCCCC)C(F)(F)F)cc1</smiles>

$\mathrm{X}=\mathrm{F}, \mathbf{3} \mathbf{i}, 86 \%$

$X=\mathrm{Cl}, 3 \mathbf{j}, 80 \%$<smiles>CCCCCCC(=CC=Cc1ccc2ccccc2c1)C(F)(F)F</smiles>

3k, $81 \%$<smiles>CCOC(/C=C/C=C/c1ccccc1)C(F)(F)F</smiles>

3 I, $83 \%$<smiles>CCCCCOC(C=CN1CCCC1=O)C(F)(F)F</smiles>

$3 \mathrm{~m}, 61 \%^{\mathrm{a}}$

Scheme 1: Palladium-catalyzed Heck-type reaction of 2-bromo-1,1,1-trifluorohexane (2a) with alkenes 1. Reaction conditions (unless otherwise specified): 1 ( $0.4 \mathrm{mmol}, 1.0$ equiv), $2 \mathrm{a}$ (2.0 equiv), DCE $(3 \mathrm{~mL}), 80^{\circ} \mathrm{C}, 16 \mathrm{~h}$. All reported yields are those of isolated products. ${ }^{\mathrm{a}}$ The reaction was conducted at $100^{\circ} \mathrm{C}$. 


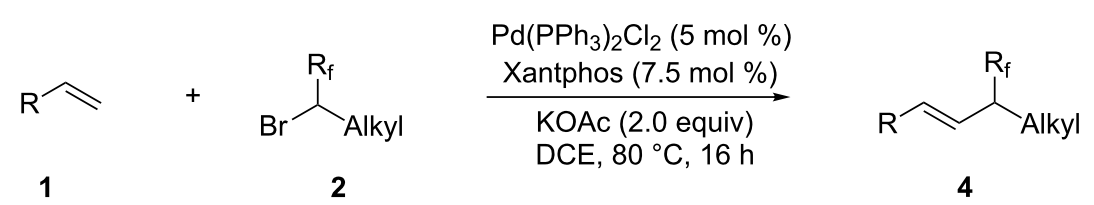<smiles>[X]c1ccc(/C=C/C(CCc2ccccc2)C(F)(F)F)cc1</smiles>

$X=$ OAc, $4 a, 92 \%$

$\mathrm{X}=\mathrm{Cl}, \mathbf{4 b}, \mathbf{9 4 \%}$<smiles>[X]c1ccc(/C=C/C(CCCO)C(F)(F)F)cc1</smiles>

$X=$ OAc, $4 e, 97 \%$

$\mathrm{X}=\mathrm{CN}, \mathbf{4 f}, 85 \%$<smiles>CC(C)(C)OCCCC(/C=C/c1ccc2ccccc2c1)C(F)(F)F</smiles>

$4 i, 91 \%$<smiles>O=CN(C=O)CCCC(/C=C/c1ccc(Cl)cc1)C(F)(F)F</smiles>

4k, $91 \%$<smiles>FC(F)(F)C(/C=C/c1ccc2ccccc2c1)CCc1ccccc1</smiles>

4c, $90 \%$<smiles>FC(F)(F)C(/C=C/C=C/c1ccccc1)CCc1ccccc1</smiles>

4d, $71 \%$<smiles>COc1ccc(/C=C/C(CCCOC(=O)OCc2ccccc2)C(F)(F)F)cc1</smiles><smiles>CC(C)(C)OCCCC(/C=C/C=C/c1ccccc1)C(F)(F)F</smiles>

4h, $80 \%$

4g, $69 \%$<smiles>O=C1c2ccccc2C(=O)N1CCCC(/C=C/c1ccc2ccccc2c1)C(F)(F)F</smiles>

$4 j, 85 \%^{a}$<smiles>[X]c1ccc(/C=C/C(CCCC)C(F)(C(=O)OCC)C(F)(F)F)cc1</smiles>

$X=\mathrm{OMe}, 4 \mathrm{I}, 82 \%^{\mathrm{b}}$

$X=F, 4 m, 76 \%^{a}$

Scheme 2: Palladium-catalyzed Heck-type reaction of fluorinated secondary bromides (iodides) 2 with alkenes 1. Reaction conditions (unless otherwise specified): $1\left(0.4 \mathrm{mmol}, 1.0\right.$ equiv), $2\left(2.0\right.$ equiv), DCE $(3 \mathrm{~mL}), 80^{\circ} \mathrm{C}, 16 \mathrm{~h}$. All reported yields are those of isolated products. ${ }^{\text {aThe reaction was }}$ carried out in $0.2 \mathrm{mmol}$ scale. ${ }^{b}$ Ethyl 2,2-difluoro-2-iodoacetate was used.

Table 2: Radical inhibition experiments of Pd-catalyzed cross-coupling between 1a and 2-bromo-1,1,1-trifluorohexane (2a) ${ }^{\mathrm{a}}$.<smiles>C=Cc1ccccc1</smiles>

1a<smiles>CCCCCC(C(C)Br)C(F)(F)F</smiles>

2a

$$
\frac{\mathrm{Pd}\left(\mathrm{PPh}_{3}\right)_{2} \mathrm{Cl}_{2}(5 \mathrm{~mol} \%)}{\text { Xantphos }(7.5 \mathrm{~mol} \%)}
$$

DCE, $80^{\circ} \mathrm{C}, 16 \mathrm{~h}$<smiles>CCCCCC(=Cc1ccccc1)C(F)(F)F</smiles>

$3 a$

\begin{tabular}{ccc}
\hline entry & additive (equiv) & 3a, yield $(\%)^{\mathrm{b}}$ \\
\hline 1 & none & $95(88)$ \\
2 & 1,4-dinitrobenzene $(0.2)$ & 22 \\
3 & 1,4-dinitrobenzene $(1.0)$ & 5 \\
4 & hydroquinone $(0.2)$ & 0 \\
\hline
\end{tabular}

aReaction conditions: 1 (0.4 mmol, 1.0 equiv), 2 a (2.0 equiv), KOAc (2.0 equiv), DCE ( $3 \mathrm{~mL}), 16 \mathrm{~h} .{ }^{\mathrm{b}}$ Determined by ${ }^{19} \mathrm{~F}$ NMR using fluorobenzene as an internal standard. 
of an electron transfer scavenger 1,4-dinitrobenzene [25-27] dramatically diminished the yield of $\mathbf{3 a}$ (Table 2, entries 2 and 3 ), and catalytic amount of radical inhibitor hydroquinone totally shut down the reaction (Table 2 , entry 4 ). Thus, these results suggest that a secondary trifluoromethylated alkyl radical via a SET pathway is likely involved in the reaction.

The existence of an alkyl radical species was further confirmed by the radical clock experiment. As illustrated in Scheme 3 , when $\alpha$-cyclopropylstyrene (5) [40] was subjected to the reaction, a ring-opened compound $\mathbf{6}$ was isolated in 55\% yield, demonstrating that a secondary trifluromethylated alkyl radical existed in the reaction is reasonable.

On the basis of these results and our previous reports [25-27], a plausible mechanism is proposed (Scheme 4). The reaction begins with the reaction of $\left[\mathrm{PdL}_{n}(0)\right]$ with secondary trifluoromethylated $\mathbf{2}$ via a SET pathway to generate alkyl radical $\mathbf{B}$. B subsequently reacts with alkene to produce new radical species $\mathbf{D}$, which then recombines with $\left[\mathrm{L}_{n} \mathrm{Pd}(\mathrm{I}) \mathrm{Br}\right] \mathbf{C}$ to give the key palladium-complex E. Finally, a $\beta$-hydride elimination delivers trifluoromethylated allylic products $\mathbf{3}$ and $\mathbf{4}$.

\section{Conclusion}

In conclusion, we have developed an efficient method for preparation of aliphatic alkenes branched with a trifluoromethyl group by palladium catalyzed Heck-type reaction of secondary trifluoromethylated alkyl bromides. The reaction proceeds under mild conditions and showed good functional group compatibility, even towards formyl and hydroxy groups, thus providing a facile route for applications in discovering biologically interesting molecules. Preliminary mechanistic studies reveal that a secondary trifluoromethylated alkyl radical is involved in the reaction.<smiles>C=C(c1ccccc1)C1[CH+]C1</smiles>

5<smiles>CCCCC(Br)C(F)(F)F</smiles>

$2 a$

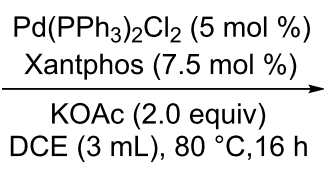

KOAc (2.0 equiv) DCE $(3 \mathrm{~mL}), 80^{\circ} \mathrm{C}, 16 \mathrm{~h}$

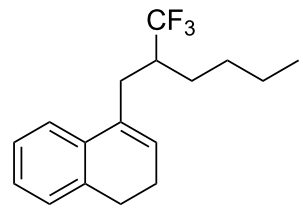

6, $55 \%$

Scheme 3: Radical clock experiment for mechanistic studies.

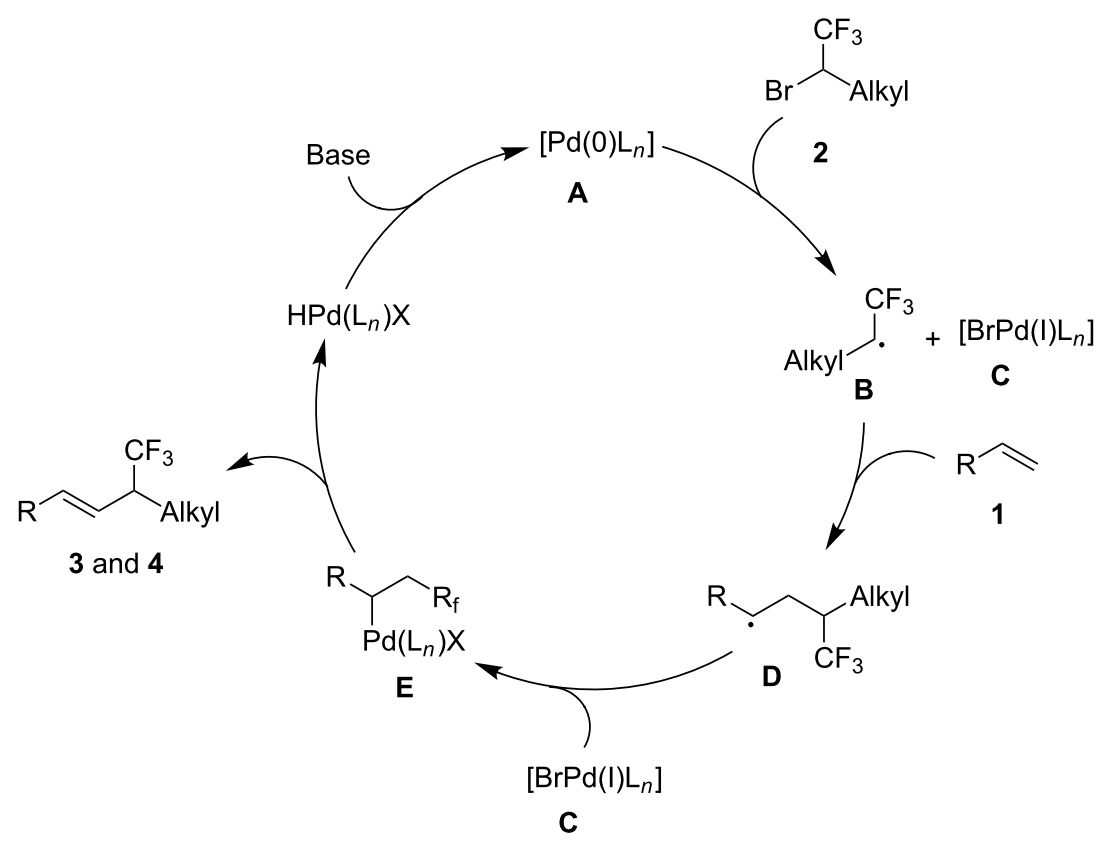




\section{Supporting Information}

\section{Supporting Information File 1}

General experimental information, experimental details on the synthesis of compounds $\mathbf{2}-\mathbf{4}$ and $\mathbf{6}$; full characterization data as well as ${ }^{1} \mathrm{H} /{ }^{19} \mathrm{~F} /{ }^{13} \mathrm{C}$ NMR spectra of all products. [http://www.beilstein-journals.org/bjoc/content/ supplementary/1860-5397-13-258-S1.pdf]

\section{Acknowledgements}

This work was financially supported by the National Natural Science Foundation of China (21425208, 21672238, 21332010, and 2141002), the Strategic Priority Research Program of the Chinese Academy of Sciences (No. XDB20000000), and Shanghai Institute of Organic Chemistry, Chinese Academy of Sciences.

\section{ORCID ${ }^{\circledR}$ iDs}

Xingang Zhang - https://orcid.org/0000-0002-4406-6533

\section{References}

1. Furuya, T.; Kamlet, A. S.; Ritter, T. Nature 2011, 473, 470. doi:10.1038/nature10108

2. Tomashenko, O. A.; Grushin, V. V. Chem. Rev. 2011, 111, 4475. doi: $10.1021 /$ cr1004293

3. Hollingworth, C.; Gouverneur, V. Chem. Commun. 2012, 48, 2929. doi:10.1039/c2cc16158c

4. Besset, T.; Schneider, C.; Cahard, D. Angew. Chem., Int. Ed. 2012, 51, 5048. doi:10.1002/anie.201201012

5. Qing, F.-L. Chin. J. Org. Chem. 2012, 32, 815. doi:10.6023/cjoc1202021

6. Wipf, P.; Henninger, T. C.; Geib, S. J. J. Org. Chem. 1998, 63, 6088. doi:10.1021/jo981057v

7. Xiao, J.; Weisblum, B.; Wipf, P. J. Am. Chem. Soc. 2005, 127, 5742. doi:10.1021/ja051002s

8. Couve-Bonnaire, S.; Cahard, D.; Pannecoucke, X. Org. Biomol. Chem. 2007, 5, 1151. doi:10.1039/b701559c

9. Narumi, T.; Hayashi, R.; Tomita, K.; Kobayashi, K.; Tanahara, N.; Ohno, H.; Naito, T.; Kodama, E.; Matsuoka, M.; Oishi, S.; Fujii, N. Org. Biomol. Chem. 2010, 8, 616. doi:10.1039/B917236J

10. Seyferth, D.; Simon, R. M.; Sepelak, D. J.; Klein, H. A. J. Org. Chem. 1980, 45, 2273. doi:10.1021/jo01299a055

11. Long, Z.-Y.; Chen, Q.-Y. J. Org. Chem. 1999, 64, 4775. doi:10.1021/j09900937

12. Murakami, S.; Ishii, H.; Fuchigami, T. J. Fluorine Chem. 2004, 125, 609. doi:10.1016/j.jluchem.2003.12.015

13. Ghattas, W.; Hess, C. R.; lacazio, G.; Hardre, R.; Klinman, J. P.; Réglier, M. J. Org. Chem. 2006, 71, 8618. doi:10.1021/jo061022s

14. Yu, C.; Iqbal, N.; Park, S.; Cho, E. J. Chem. Commun. 2014, 50, 12884. doi:10.1039/C4CC05467A

15. Besset, T.; Poisson, T.; Pannecoucke, X. Chem. - Eur. J. 2014, 20, 16830. doi:10.1002/chem.201404537

16. Hu, C.-M.; Quu, Y.-L.; Qin, F.-L. J. Fluorine Chem. 1991, 51, 295. doi:10.1016/S0022-1139(00)80300-0
17. Umemoto, T.; Kuriu, Y.; Miyano, O. Tetrahedron Lett. 1982, 23, 3579. doi:10.1016/S0040-4039(00)87675-3

18. Taguchi, T.; Kitagawa, O.; Morikawa, T.; Nishiwaki, T.; Uehara, H.; Endo, H.; Kobayashi, Y. Tetrahedron Lett. 1986, 27, 6103. doi:10.1016/S0040-4039(00)85409-X

19. Yokomatsu, T.; Suemune, K.; Murano, T.; Shibuya, S. J. Org. Chem. 1996, 61, 7207. doi:10.1021/jo960896j

20. Sato, K.; Kawata, R.; Ama, F.; Omate, M.; Ando, A.; Kumadaki, I. Chem. Pharm. Bull. 1999, 47, 1013. doi:10.1248/cpb.47.1013

21. Schwaebe, M. K.; McCarthy, J. R.; Whitten, J. P. Tetrahedron Lett. 2000, 41, 791. doi:10.1016/S0040-4039(99)02212-1

22. Chu, L.; Qing, F.-L. Org. Lett. 2010, 12, 5060. doi:10.1021/ol1023135

23. Fier, P. S.; Hartwig, J. F. J. Am. Chem. Soc. 2012, 134, 5524. doi:10.1021/ja301013h

24. Prakash, G. K. S.; Ganesh, S. K.; Jones, J.-P.; Kulkarni, A.; Masood, K.; Swabeck, J. K.; Olah, G. A. Angew. Chem., Int. Ed. 2012 , 51, 12090. doi:10.1002/anie.201205850

25. Feng, Z.; Min, Q.-Q.; Zhao, H.-Y.; Gu, J.-W.; Zhang, X. Angew. Chem., Int. Ed. 2015, 54, 1270. doi:10.1002/anie.201409617

26. Feng, Z.; Min, Q.-Q.; Zhang, X. Synthesis 2015, 47, 2912. doi:10.1055/s-0035-1560457

27. Feng, Z.; Xiao, Y.-L.; Zhang, X. Org. Chem. Front. 2016, 3, 466. doi:10.1039/C6Q000005C

28. Firmansjah, L.; Fu, G. C. J. Am. Chem. Soc. 2007, 129, 11340. doi:10.1021/ja075245r

29. Bloome, K. S.; McMahen, R. L.; Alexanian, E. J. J. Am. Chem. Soc. 2011, 133, 20146. doi:10.1021/ja2091883

30. McMahon, C. M.; Alexanian, E. J. Angew. Chem., Int. Ed. 2014, 53, 5974. doi:10.1002/anie.201311323

31. Zou, Y.; Zhou, S. Chem. Commun. 2014, 50, 3725. doi:10.1039/C4CC00297K

32. Okano, T.; Sugiura, H.; Fumoto, M.; Matsubara, H.; Kusukawa, T.; Fujita, M. J. Fluorine Chem. 2002, 114, 91. doi:10.1016/S0022-1139(02)00002-7

33. Stille, J. K. In The Chemistry of the Metal-Carbon Bond; Patai, S., Ed.; chap. 9, Vol. 2; Wiley: New York, 1985; pp 625-787.

34. Ozawa, F.; Ito, T.; Yamamoto, A. J. Am. Chem. Soc. 1980, 102, 6457. doi:10.1021/ja00541a013

35. Hartwig, J. F. Organotransition Metal Chemistry: From Bonding to Catalysis; Chapter 10; University Science Books: Sausalito, CA, 2009; pp 397-416.

36. Taguchi, T.; Namba, R.; Nakazawa, M.; Nakajima, M.; Nakama, Y.; Kobayashi, Y.; Hara, N.; Ikekawa, N. Tetrahedron Lett. 1988, 29, 227. doi:10.1016/S0040-4039(00)80061-1

37. Jiang, B.; Lu, Y.; Zhou, W.-s. J. Org. Chem. 2000, 65, 6231. doi:10.1021/jo000059o

38. Grushin, V. V.; Marshall, W. J. J. Am. Chem. Soc. 2006, 128, 12644. doi:10.1021/ja064935c

39. Bakhmutov, V. I.; Bozoglian, F.; Gómez, K.; González, G.; Grushin, V. V.; Macgregor, S. A.; Martin, E.; Miloserdov, F. M.; Novikov, M. A.; Panetier, J. A.; Romashov, L. V. Organometallics 2012, 31, 1315. doi:10.1021/om200985g

40. Baldwin, J. E. Chem. Rev. 2003, 103, 1197. doi:10.1021/cr010020z 


\section{License and Terms}

This is an Open Access article under the terms of the Creative Commons Attribution License

(http://creativecommons.org/licenses/by/4.0), which permits unrestricted use, distribution, and reproduction in any medium, provided the original work is properly cited.

The license is subject to the Beilstein Journal of Organic Chemistry terms and conditions:

(http://www.beilstein-journals.org/bjoc)

The definitive version of this article is the electronic one which can be found at:

doi:10.3762/bjoc. 13.258 